\title{
Application of the Least Squares Method in Axisymmetric Biharmonic Problems
}

\author{
Vasyl Chekurin and Lesya Postolaki \\ Pidstryhach Institute for Applied Problems of Mechanics and Mathematics of National Academy of Sciences of Ukraine, \\ 3-b Naukova Street, Lviv 79060, Ukraine
}

Correspondence should be addressed to Vasyl Chekurin; v.chekurin@gmail.com

Received 29 March 2016; Revised 29 May 2016; Accepted 2 June 2016

Academic Editor: Masoud Hajarian

Copyright (C) 2016 V. Chekurin and L. Postolaki. This is an open access article distributed under the Creative Commons Attribution License, which permits unrestricted use, distribution, and reproduction in any medium, provided the original work is properly cited.

\begin{abstract}
An approach for solving of the axisymmetric biharmonic boundary value problems for semi-infinite cylindrical domain was developed in the paper. On the lateral surface of the domain homogeneous Neumann boundary conditions are prescribed. On the remaining part of the domain's boundary four different biharmonic boundary pieces of data are considered. To solve the formulated biharmonic problems the method of least squares on the boundary combined with the method of homogeneous solutions was used. That enabled reducing the problems to infinite systems of linear algebraic equations which can be solved with the use of reduction method. Convergence of the solution obtained with developed approach was studied numerically on some characteristic examples. The developed approach can be used particularly to solve axisymmetric elasticity problems for cylindrical bodies, the heights of which are equal to or exceed their diameters, when on their lateral surface normal and tangential tractions are prescribed and on the cylinder's end faces various types of boundary conditions in stresses in displacements or mixed ones are given.
\end{abstract}

\section{Introduction}

Many practically important problems bring the biharmonic equation

$$
\begin{aligned}
\Delta^{2} w & =0, \\
w & =w(x), \quad x \in S \subset R^{2}
\end{aligned}
$$

considering in $2 \mathrm{D}$ domains $S$ with Lipschitz-continuous boundary $\partial S$.

Here $\Delta$ is Laplace differential operator: $\Delta=\nabla \cdot \nabla$, where $\nabla$ stands for the gradient operator in $R^{2}$ and the dot (.) denotes scalar product. Function $w$ is considered as four-time differentiable one in $S$.

The classical formulations of biharmonic problems distinguish the Dirichlet and Neumann boundary value problems. Two kinds of Dirichlet problems are usually considered for biharmonic equation (1). In the problem of the first and second kinds the biharmonic functions should be subordinated to boundary conditions (2) and (3) correspondingly [1]:

$$
\begin{aligned}
&\left.w\right|_{x \in \partial S}=f_{0}(x), \\
&\left.\frac{\partial w}{\partial \nu}\right|_{x \in \partial S}= f_{1}(x), \\
& x \in \partial S, \\
&\left.w\right|_{x \in \partial S}=f_{0}(x), \\
&\left.\Delta w\right|_{x \in \partial S}=f_{1}(x), \quad \\
& x \in \partial S .
\end{aligned}
$$

Here $\partial / \partial \nu \equiv \nu \cdot \nabla$ is the operator of normal derivative on $\partial S, v$ is the outward unit normal vector to $\partial S$, and $f_{0}(x)$ and $f_{1}(x)$ are given functions. 
Various kinds of Neumann problems for (1) can also be considered $[2,3]$. In simplest cases these are the problems with boundary conditions (4), (5), or (6):

$$
\begin{aligned}
& \left.\frac{\partial w}{\partial \nu}\right|_{x \in \partial S}=f_{0}(x), \\
& \left.\frac{\partial^{2} w}{\partial \nu^{2}}\right|_{x \in \partial S}=f_{1}(x), \\
& \left.\frac{\partial w}{\partial \nu}\right|_{x \in \partial S}=f_{0}(x), \\
& \left.\frac{\partial \Delta w}{\partial v}\right|_{x \in \partial S}=f_{1}(x), \\
& \left.\frac{\left.\partial w\right|_{x \in \partial S}}{d \Delta w}\right|_{x \in \partial S}=f_{0}(x), \\
& x \in \partial S,
\end{aligned}
$$

One can distinguish two kinds of biharmonic mixed problems $[4,5]$. In the mixed problem of the first kind (1) is considered subject to boundary conditions which are weighted combinations of Dirichlet boundary conditions and Neumann boundary conditions (so-called Robin boundary condition). In another case Dirichlet data are prescribed on one part of the boundary and Neumann data are prescribed on the remainder.

Various methods are used for solving of the biharmonic problems. Among them are iterative methods [6], boundary integral method [7], method of finite differences [8], finite element method [9], and so forth.

Significant interest in biharmonic problems in rectangle arises in 2D theory of elasticity [10]. In this connection we should refer to the so-called method of homogeneous solutions [11-14] used for these problems' solving. An idea of the method consists in representing the solution as a series expansion in some complete system of biharmonic functions being solutions of a homogeneous biharmonic problem on infinite strip [11, 14]. These functions satisfy homogeneous Neumann-type boundary conditions on the strip's sides. As such representation automatically satisfies (1) and homogeneous boundary conditions on two opposite sides of the rectangle, to find the solution it is necessary to determine the expansion coefficients by subordinating the solution to the boundary data prescribed on the other two opposite rectangle's sides. To do that the least squares method was applied in [14]. In such way the problem was reduced to a problem of nonconstrained optimization. The approach was applied to solve Neumann problem and some mixed biharmonic problems on rectangle.

The method of least squares on the boundary was also used in [15] to solve the first Dirichlet problem on rectangle. Here the solution of the biharmonic equation was presented as a linear combination of finite system of biharmonic polynomials.

In [16] an axisymmetric biharmonic problem for a finite cylindrical domain was considered. The solution was presented there as the Fourier-Bessel expansion. In [17] the method of fundamental solution was applied to solve axisymmetric Dirichlet biharmonic problem (1) and (2).

In this paper we consider the method of least squares on the boundary combined with the method of homogeneous solutions in application to axisymmetric biharmonic problems for a semi-infinite cylindrical domain.

\section{Problem Formulation}

Problems of elastic equilibrium in axisymmetric case can be reduced to axisymmetric biharmonic equation (1), where $\Delta=\partial^{2} / \partial r^{2}+(1 / r)(\partial / \partial r)+\partial^{2} / \partial z^{2}$ is axisymmetric Laplace operator in cylindrical coordinate ( $r$ and $z$ stand for radial and axial coordinates).

Biharmonic function $w$ in this case has the sense of Love stress function, through which displacement components $u_{r}, u_{z}$ and stress components $\sigma_{r r}, \sigma_{r z}, \sigma_{\theta \theta}, \sigma_{z z}$ can be expressed as [10]

$$
\begin{aligned}
u_{r} & =-\frac{\partial^{2} w}{\partial r \partial z}, \\
u_{z} & =\frac{\partial^{2} w}{\partial z^{2}}+2(1-v) \nabla^{2} w, \\
\frac{1}{2 \mu} \sigma_{r r} & =\frac{\partial}{\partial z}\left(\nu \nabla^{2} w-\frac{\partial^{2} w}{\partial r^{2}}\right), \\
\frac{1}{2 \mu} \sigma_{r z} & =\frac{\partial}{\partial r}\left((1-\nu) \nabla^{2} w-\frac{\partial^{2} w}{\partial z^{2}}\right), \\
\frac{1}{2 \mu} \sigma_{z z} & =\frac{\partial}{\partial z}\left((2-\nu) \nabla^{2} w-\frac{\partial^{2} w}{\partial z^{2}}\right), \\
\frac{1}{2 \mu} \sigma_{\theta \theta} & =\frac{\partial}{\partial z}\left[\nu \nabla^{2} w-\frac{1}{r} \frac{\partial w}{\partial r}\right],
\end{aligned}
$$

where $\nu \in[0,0.5]$ is Poisson ratio and $\mu$ stands for shear modulus.

We will consider four biharmonic problems for semiinfinite cylindrical domain $\mathbf{V}=(0 \leq r<1,0<\theta \leq 2 \pi, 0<$ $z<\infty)$ with prescribed stresses $\sigma_{r r}$ and $\sigma_{r z}$ on its lateral surface $\mathbf{D}=(r=1,0<\theta \leq 2 \pi, 0<z<\infty)$ :

$$
\begin{gathered}
\left.\sigma_{r r}\right|_{r=1}=f_{1}(z), \\
\left.\sigma_{r z}\right|_{r=1}=f_{2}(z) .
\end{gathered}
$$

Here $f_{1}(z)$ and $f_{2}(z)$ are integrable functions which decay when $z$ tends to infinity. 
Problems I to IV are distinguished by boundary conditions prescribed on the plane circular area $\mathbf{S}=(0 \leq r<1,0<$ $\theta \leq 2 \pi, z=0)$.

Problem I. Consider the following:

$$
\begin{gathered}
\left.\sigma_{z z}\right|_{z=0}=\varphi_{1}(r), \\
\left.\sigma_{r z}\right|_{z=0}=\varphi_{2}(r) .
\end{gathered}
$$

Problem II. Consider the following:

$$
\begin{aligned}
& \left.u_{z}\right|_{z=0}=\varphi_{3}(r), \\
& \left.u_{r}\right|_{z=0}=\varphi_{4}(r) .
\end{aligned}
$$

Problem III. Consider the following:

$$
\begin{gathered}
\left.\sigma_{z z}\right|_{z=0}=\varphi_{1}(r), \\
\left.u_{r}\right|_{z=0}=\varphi_{4}(r) .
\end{gathered}
$$

Problem IV. Consider the following:

$$
\begin{gathered}
\left.u_{z}\right|_{z=0}=\varphi_{3}(r), \\
\left.\sigma_{r z}\right|_{z=0}=\varphi_{2}(r),
\end{gathered}
$$

where $\varphi_{1}(r), \varphi_{2}(r), \varphi_{3}(r)$, and $\varphi_{4}(r)$ are given function.

With the use of relations (7) and (8) we can express the boundary conditions (9)-(13) in terms of function $w(r, z)$. We can see that problem I is of Neumann type. It is solvable only if the functions $\varphi_{1}(r)$ and $f_{2}(z)$ satisfy the condition

$$
\int_{0}^{1} r \varphi(r) d r-2 \pi \int_{0}^{\infty} f_{2}(z) d z=0 .
$$

Problems III and IV should be classified as mixed ones.

To use the method of homogeneous solutions we reduce the problems to corresponding problems with homogeneous boundary conditions on $\mathbf{D}$. To do that we consider an auxiliary biharmonic problem for infinite cylindrical domain $\mathbf{V}^{0}=(0 \leq r<1,0<\theta \leq 2 \pi,-\infty<z<\infty)$ with boundary conditions

$$
\begin{aligned}
& \left.\sigma_{r r}^{0}\right|_{r=1}=f_{1}^{0}(z), \\
& \left.\sigma_{r z}^{0}\right|_{r=1}=f_{2}^{0}(z),
\end{aligned}
$$

where $f_{1}^{0}(z)$ and $f_{2}^{0}(z)$ are defined on the line $z \in(-\infty, \infty)$ integrable functions that satisfy conditions $f_{1}^{0}(z)=f_{1}(z)$, $f_{2}^{0}(z)=f_{2}(z)$, for $z \geq 0$, and both decay when $z \rightarrow-\infty$. For instance, we can choose $f_{1}^{0}(z)$ and $f_{2}^{0}(z)$ as

$$
\begin{aligned}
& f_{1}^{0}(z)= \begin{cases}f_{1}(z), & z \geq 0, \\
f_{1}(-z), & z<0,\end{cases} \\
& f_{2}^{0}(z)= \begin{cases}f_{2}(z), & z \geq 0, \\
-f_{2}(-z), & z<0,\end{cases}
\end{aligned}
$$

or as

$$
\begin{aligned}
& f_{1}^{0}(z)= \begin{cases}f_{1}(z), & z \geq 0, \\
-f_{1}(-z), & z<0,\end{cases} \\
& f_{2}^{0}(z)= \begin{cases}f_{2}(z), & z \geq 0, \\
f_{2}(-z), & z<0 .\end{cases}
\end{aligned}
$$

Problem (1) and (15) was solved with the use of the Fourier integral transform. One can find the solution in [18].

Let $v^{0}(r, z)$ be the solution of problem (1) and (15) and $u_{r}^{0}, u_{z}^{0}$ and $\sigma_{r r}^{0}, \sigma_{r z}^{0}, \sigma_{\theta \theta}^{0}, \sigma_{z z}^{0}$ the functions, calculated due to formulas (7) and (8) correspondingly for solution $v^{0}(r, z)$. We introduce the functions

$$
\begin{aligned}
& \left.\varphi_{1}^{0}(r) \equiv \sigma_{z z}^{0}\right|_{z=0}, \\
& \left.\varphi_{2}^{0}(r) \equiv \sigma_{r z}^{0}\right|_{z=0}, \\
& \left.\varphi_{3}^{0}(r) \equiv u_{z}^{0}\right|_{z=0}, \\
& \left.\varphi_{4}^{0}(r) \equiv u_{r}^{0}\right|_{z=0},
\end{aligned}
$$

Now the solutions of the biharmonic problems I to IV can be presented as $w=v^{0}+v$, where $v(r, z)$ is the solution of the biharmonic problems for domain $\mathbf{V}$ on the lateral surface D $\subset \partial \mathbf{V}$ of which Neumann's homogeneous conditions are prescribed:

$$
\begin{array}{r}
\left.\frac{\partial}{\partial z}\left(v \Delta v-\frac{\partial^{2} v}{\partial r^{2}}\right)\right|_{r=1}=0 \\
\left.\frac{\partial}{\partial r}\left((1-v) \Delta v-\frac{\partial^{2} v}{\partial z^{2}}\right)\right|_{r=1}=0
\end{array}
$$

On the surface $\mathbf{S} \subset \partial \mathbf{V}$ the function $v(r, z)$ obeys one pair of conditions (20)-(23):

$$
\begin{array}{r}
\left.\frac{\partial}{\partial z}\left((2-v) \Delta v-\frac{\partial^{2} v}{\partial z^{2}}\right)\right|_{z=0}=\sigma(r), \\
\left.\frac{\partial}{\partial r}\left((1-v) \Delta v-\frac{\partial^{2} v}{\partial z^{2}}\right)\right|_{z=0}=\tau(r), \\
\left.\left(\frac{\partial^{2} v}{\partial z^{2}}+2(1-v) \Delta v\right)\right|_{z=0}=n(r), \\
-\left.\frac{\partial^{2} v}{\partial r \partial z}\right|_{z=0}=t(r),
\end{array}
$$




$$
\begin{array}{r}
\left.\frac{\partial}{\partial z}\left((2-v) \Delta v-\frac{\partial^{2} v}{\partial z^{2}}\right)\right|_{z=0}=\sigma(r), \\
-\left.\frac{\partial^{2} v}{\partial r \partial z}\right|_{z=0}=t(r), \\
\left.\left(\frac{\partial^{2} v}{\partial z^{2}}+2(1-v) \Delta v\right)\right|_{z=0}=n(r), \\
\left.\frac{\partial}{\partial r}\left((1-v) \Delta v-\frac{\partial^{2} v}{\partial z^{2}}\right)\right|_{z=0}=\tau(r) .
\end{array}
$$

Here

$$
\begin{gathered}
\sigma(r)=\varphi_{1}(r)-\varphi_{1}^{0}(r), \\
\tau(r)=\varphi_{2}(r)-\varphi_{2}^{0}(r), \\
n(r)=\varphi_{3}(r)-\varphi_{3}^{0}(r), \\
t(r)=\varphi_{4}(r)-\varphi_{4}^{0}(r) .
\end{gathered}
$$

So, biharmonic problems I to IV reduced to biharmonic problems (problems I' to IV') with homogeneous conditions (19) on the cylinder lateral surface and corresponding nonhomogeneous conditions (20)-(23) on the circular area $\mathbf{S}$. We will solve these problems using the method of homogeneous solution.

\section{Systems of Homogeneous Solutions in Cylindrical Coordinates}

We look for a solution of biharmonic equation (1) in the form

$$
v(r, z)=\exp (-\gamma z) f(r)
$$

Substituting (25) into (1) brings the next ordinary differential equation for the radial function $f(r)$ :

$$
\begin{aligned}
f^{\mathrm{IV}}(r) & +\frac{2}{r} f^{\prime \prime \prime}(r)+\left(2 \gamma^{2}-\frac{1}{r^{2}}\right) f^{\prime \prime}(r) \\
+ & \left(\frac{1}{r^{3}}+\frac{2}{r} \gamma^{2}\right) f^{\prime}(r)+\gamma^{4} f(r)=0 .
\end{aligned}
$$

Due to relations (19) and (25) the radial function $f(r)$ obeys at $r=1$ the boundary conditions:

$$
\begin{aligned}
& \left.\left((\nu-1) \frac{\partial^{2} f(r)}{\partial r^{2}}+v\left(\frac{1}{r} \frac{\partial f(r)}{\partial r}+\gamma^{2} f(r)\right)\right)\right|_{r=1}=0 \\
& \left((1-v)\left(\frac{\partial^{3} f(r)}{\partial r^{3}}+\frac{1}{r} \frac{\partial^{2} f(r)}{\partial r^{2}}\right)\right. \\
& \left.+\left(\frac{(\nu-1)}{r^{2}}-v \gamma^{2}\right) \frac{\partial s(r)}{\partial r}\right)\left.\right|_{r=1}=0 .
\end{aligned}
$$

The function $f(r)$ and its derivative should be finite at $r=0$.
The general solution of (26) is

$$
\begin{aligned}
& f(r)=A r J_{1}(\gamma r)+B r Y_{1}(\gamma r)+C r Y_{0}(\gamma r) \\
& \cdot\left(-J_{1}(\gamma r) Y_{0}(\gamma r)+Y_{1}(\gamma r) J_{0}(\gamma r)\right) \\
& +\operatorname{Dr}\left(-J_{1}(\gamma r) J_{0}(\gamma r) Y_{0}(\gamma r)-Y_{1}(\gamma r)\right. \\
& \left.\quad+Y_{1}(\gamma r)\left(J_{0}(\gamma r)\right)^{2}\right) .
\end{aligned}
$$

Here $A, B, C$, and $D$ stand for arbitrary constants, $J_{0}, J_{1}$ and $Y_{0}, Y_{1}$ are Bessel and Hankel functions of orders zero and one correspondingly.

To provide finiteness of solution (28) at the point $r=0$ we put $C=0, D=B$. Then, with accounting of the property of Bessel functions

$$
J_{0}(\gamma) Y_{1}(\gamma)-J_{1}(\gamma) Y_{0}(\gamma)=-\frac{2}{\pi \gamma},
$$

the radial function $f(r)$ takes the form

$$
f(r)=r J_{1}(\gamma r) A-\frac{2}{\pi \gamma} J_{0}(\gamma r) B .
$$

Substitution of (30) into boundary conditions (27) brings the linear homogeneous system regarding the constants $A$ and $B$ :

$$
\begin{aligned}
& \pi \gamma\left((1-2 \gamma) J_{0}(\gamma)-\gamma J_{1}(\gamma)\right) A \\
& \quad+2\left(\gamma J_{0}(\gamma)-J_{1}(\gamma)\right) B=0, \\
& \pi\left(\gamma J_{0}(\gamma)+2(1-\gamma) J_{1}(\gamma)\right) A+2 J_{1}(\gamma) B=0 .
\end{aligned}
$$

Nontrivial solutions of system (31) exist under the condition

$$
\gamma^{2}\left(J_{0}^{2}(\gamma)+J_{1}^{2}(\gamma)\right)+(2 \nu-2) J_{1}^{2}(\gamma)=0
$$

Transcendental equation (32) does not have any real roots except the doubly degenerate root $\gamma=0$. It does not have imaginary roots too. Hence complex roots should be considered. The set of roots of (32) contains four infinite sequences of complex roots [10]:

$$
\begin{aligned}
& \gamma_{k}^{(1)}=\alpha_{k}+i \beta_{k}, \\
& \gamma_{k}^{(2)}=\alpha_{k}-i \beta_{k}, \\
& \gamma_{k}^{(3)}=-\alpha_{k}+i \beta_{k}, \\
& \gamma_{k}^{(4)}=-\alpha_{k}-i \beta_{k}, \\
& \quad k=1,2, \ldots
\end{aligned}
$$

The values of first 15 roots of (32) are presented in Table 1: $\alpha_{k}=\left|\operatorname{Re}\left(\gamma_{k}^{(\lambda)}\right)\right| ; \beta_{k}=\left|\operatorname{Im}\left(\gamma_{k}^{(\lambda)}\right)\right| \lambda=\overline{1,4}$. The data were obtained by numerical solving of (32) at $\nu=0.25$.

Further we will use the sequences $G^{(1)}=\left\{\gamma_{k}^{(1)}, k=\right.$ $1,2, \ldots\}$ and $G^{(2)}=\left\{\gamma_{k}^{(2)}, k=1,2, \ldots\right\}$, the members of which 
TABLE 1: Real and imaginary parts of roots $\gamma_{k}^{(\lambda)}$.

\begin{tabular}{cccccccc}
\hline$k$ & $\alpha_{k}$ & $\beta_{k}$ & $k$ & $\alpha_{k}$ & $\beta_{k}$ & $k$ & $\alpha_{k}$ \\
\hline 1 & 2.69765 & 1.36735 & 6 & 18.75905 & 2.16604 & 11 & 34.50379 \\
2 & 6.05122 & 1.63814 & 7 & 21.91184 & 2.24211 & 12 & 37.64928 \\
3 & 9.26127 & 1.82853 & 8 & 25.06203 & 2.30817 & 13 & 40.79422 \\
4 & 12.43844 & 1.96742 & 9 & 28.21044 & 2.36656 & 14 & 43.93871 \\
5 & 15.60220 & 2.07642 & 10 & 31.35758 & 2.41886 & 15 & 47.08284 \\
\hline
\end{tabular}

have positive real parts. That provides finiteness of solution (25) at infinity $z \rightarrow \infty$.

The set of solutions of linear system (31) is

$$
\begin{aligned}
& A_{k}=\kappa_{k} B_{k}, \\
& \kappa_{k}=\frac{2 J_{1}\left(\gamma_{k}\right)}{\pi\left((2 \nu-2) J_{1}\left(\gamma_{k}\right)-\gamma_{k} J_{0}\left(\gamma_{k}\right)\right)},
\end{aligned}
$$

where $B_{k}$ are indefinite complex constants. The notations $\kappa_{k}^{(1)}=\kappa, \kappa_{k}^{(2)}=\bar{\kappa}$ are used in (34) (the overline means complex conjugation).

With this in view we obtain two infinite sequences $\mathbf{R}^{(1)}=$ $\left\{f_{k}^{(1)}, k=1,2, \ldots\right\}$ and $\mathbf{R}^{(2)}=\left\{f_{k}^{(2)}, k=1,2, \ldots\right\}$ of radial functions

$$
\begin{aligned}
& f_{k}^{(1)}(r)=\left(r J_{1}\left(\gamma_{k}^{(1)} r\right) \kappa_{k}^{(1)}-\frac{2}{\pi \gamma_{k}} J_{0}\left(\gamma_{k}^{(1)} r\right)\right), \\
& f_{k}^{(2)}(r)=\left(r J_{1}\left(\gamma_{k}^{(2)} r\right) \kappa_{k}^{(2)}-\frac{2}{\pi \gamma_{k}} J_{0}\left(\gamma_{k}^{(2)} r\right)\right)
\end{aligned}
$$

and two infinite sequences $V^{(1)}=\left\{v_{k}^{(1)}, k=1,2, \ldots\right\}$ and $V^{(2)}=\left\{v_{k}^{(2)}, k=1,2, \ldots\right\}$ homogeneous solutions

$$
\begin{aligned}
& v_{k}^{(1)}(r, z)=f_{k}^{(1)}(r) \exp \left(-\gamma_{k}^{(1)} z\right), \\
& v_{k}^{(2)}(r, z)=f_{k}^{(2)}(r) \exp \left(-\gamma_{k}^{(2)} z\right) .
\end{aligned}
$$

In Figures 1 and 2 the real and imaginary parts of functions $f_{k}^{(1)}(r)$ are shown for $k=1,2,3,4,5$ (curves 1, 2, 3,4 , and 5 correspondingly).

As the functions $f_{k}^{(1)}(r)$ and $f_{k}^{(2)}(r)$ are solutions of homogeneous boundary value problem (26) and (27) both sequences $\mathbf{R}^{(1)}$ and $\mathbf{R}^{(2)}$ form independent functional bases on the segment $r \in[0,1]$ in complex domain. Their real $\operatorname{Re}\left(f_{k}^{(1)}(r)\right)$ and $\operatorname{Im}\left(f_{k}^{(1)}(r)\right)$ parts form independent functional bases on the same segment in real domain.

We will use the two sequences $V^{(1)}$ and $V^{(2)}$ of complex valued functions (36) to construct the real solutions for four biharmonic problems in $\mathbf{V}$ (problems $\mathrm{I}^{\prime}$ to $\mathrm{IV}^{\prime}$ ) prescribed on $\mathbf{S} \subset \partial \mathbf{V}$ boundary conditions given by formulas (20) to (23) correspondingly. On the remining part $\mathbf{D}$ of $\partial \mathbf{V}$ homogeneous conditions (19) are prescribed for all problems.

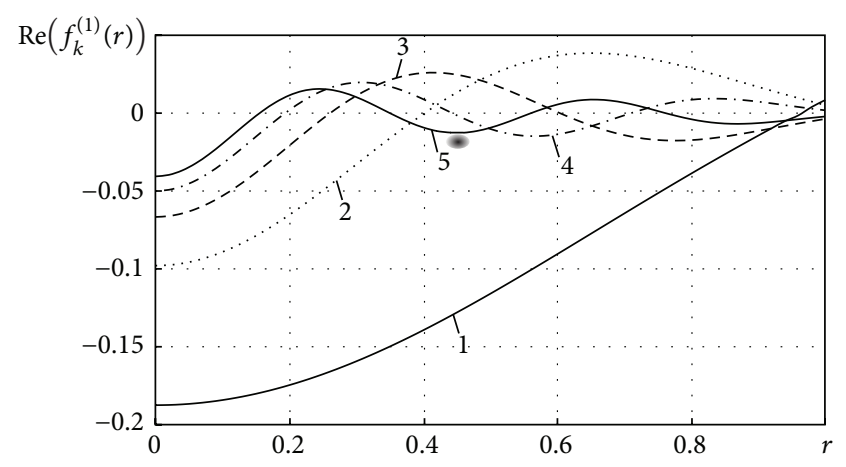

FIGURE 1: Real parts of function $f_{k}^{(1)}(r)$.

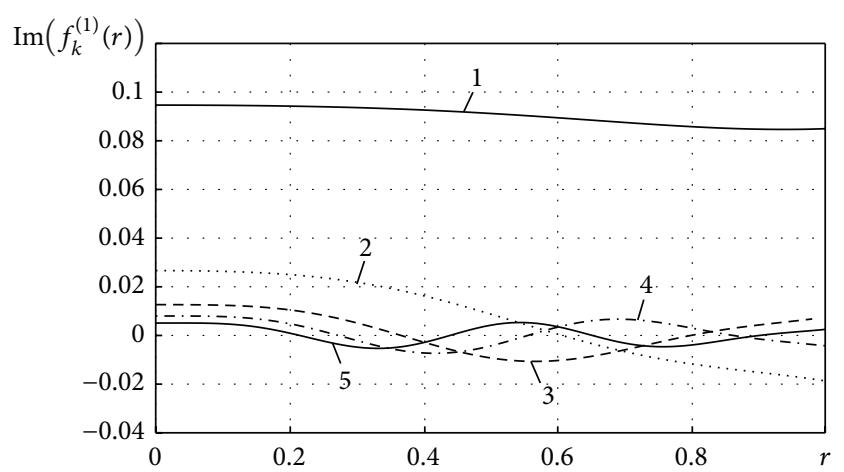

FIGURE 2: Imaginary parts of function $f_{k}^{(1)}(r)$.

As the sequences $v_{k}^{(1)}(r, z)$ and $v_{k}^{(2)}(r, z)$ are mutually complex-conjugated we present the solution $v(r, z)$ in the form

$$
v(r, z)=\frac{1}{2} \sum_{k=1}^{\infty} \sum_{p=1}^{2} B_{k}^{(p)} v_{k}^{(p)}(r, z),
$$

where $B_{k}^{(1)}, B_{k}^{(2)} \equiv \overline{B_{k}^{(1)}}$ are indefinite complex constants.

As all functions $v_{k}^{(p)}(r, z), p=1,2$ satisfy (1) and boundary conditions (19) to solve the problems it is necessary to determine the constants $B_{k}^{(1)}, B_{k}^{(2)}$ by subordinating the solution (37) to the boundary conditions (20)-(23). 


\section{A Variational Method}

Substitution of solution (37) into boundary conditions (20)(23) brings two functional relations for each problem:

$$
\begin{aligned}
& \frac{1}{2} \sum_{k=1}^{\infty} \sum_{p=1}^{2} B_{k}^{(p)} \sigma_{k}^{(p)}=\sigma(r), \\
& \frac{1}{2} \sum_{k=1}^{\infty} \sum_{p=1}^{2} B_{k}^{(p)} \tau_{k}^{(p)}=\tau(r), \\
& \frac{1}{2} \sum_{k=1}^{\infty} \sum_{p=1}^{2} B_{k}^{(p)} n_{k}^{(p)}=n(r), \\
& \frac{1}{2} \sum_{k=1}^{\infty} \sum_{p=1}^{2} B_{k}^{(p)} t_{k}^{(p)}=t(r), \\
& \frac{1}{2} \sum_{k=1}^{\infty} \sum_{p=1}^{2} B_{k}^{(p)} \sigma_{k}^{(p)}=\sigma(r), \\
& \frac{1}{2} \sum_{k=1}^{\infty} \sum_{p=1}^{2} B_{k}^{(p)} t_{k}^{(p)}=t(r), \\
& \frac{1}{2} \sum_{k=1}^{\infty} \sum_{p=1}^{2} B_{k}^{(p)} n_{k}^{(p)}=n(r), \\
& \frac{1}{2} \sum_{k=1}^{\infty} \sum_{p=1}^{2} B_{k}^{(p)} \tau_{k}^{(p)}=\tau(r),
\end{aligned}
$$

where the following notations are used:

$$
\begin{aligned}
& \sigma_{k}^{(p)}(r)=(\nu-2) \gamma_{k}^{(p)}\left(\frac{\partial^{2} f_{k}^{(p)}(r)}{\partial r^{2}}+\frac{1}{r} \frac{\partial f_{k}^{(p)}(r)}{\partial r}\right) \\
&+(\nu-1)\left(\gamma_{k}^{(p)}\right)^{3} f_{k}^{(p)}(r), \\
& \tau_{k}^{(p)}(r)=(1-v)\left(\frac{\partial^{3} f_{k}^{(p)}(r)}{\partial r^{3}}+\frac{1}{r} \frac{\partial^{2} f_{k}^{(p)}(r)}{\partial r^{2}}\right) \\
&+\left(\frac{\nu-1}{r^{2}}-v\left(\gamma_{k}^{(p)}\right)^{2}\right) \frac{\partial f_{k}^{(p)}(r)}{\partial r}, \\
& n_{k}^{(p)}(r)=2(1-v)\left(\frac{\partial^{2} f_{k}^{(p)}(r)}{\partial r^{2}}+\frac{1}{r} \frac{\partial f_{k}^{(p)}(r)}{\partial r}\right) \\
&+(3-2 \nu)\left(\gamma_{k}^{(p)}\right)^{2} f_{k}^{(p)}(r), \\
& t_{k}^{(p)}(r)=-\gamma_{k}^{(p)} \frac{\partial f_{k}^{(p)}(r)}{\partial r} .
\end{aligned}
$$

Hence, to solve any of problems I' to IV' we should find the infinite sequences $\mathbf{B}^{(1)}=\left\{B_{k}^{(1)}, k=1,2, \ldots\right\}$ and $\mathbf{B}^{(2)}=$ $\left\{\overline{B_{k}^{(1)}}, k=1,2, \ldots\right\}$ of complex numbers the members $B_{k}^{(1)} \in$ $\mathbf{B}^{(1)}$ and $B_{k}^{(2)} \in \mathbf{B}^{(2)}$ of which transform the corresponding pair of functional equations (38) to (41) into identities. We solve the problem of determination of the sequences $\mathbf{B}^{(1)}$ and $\mathbf{B}^{(2)}$ in quadratic norm $L^{2}$ exploiting the least squares method. To do that we define the functional for each problem

$$
\begin{aligned}
F_{\mathrm{I}} & =\int_{0}^{1}\left[\left(\frac{1}{2} \sum_{k=1}^{\infty} \sum_{p=1}^{2} B_{k}^{(p)} \sigma_{k}^{(p)}-\sigma(r)\right)^{2}\right. \\
& \left.+\left(\frac{1}{2} \sum_{k=1}^{\infty} \sum_{p=1}^{2} B_{k}^{(p)} \tau_{k}^{(p)}-\tau(r)\right)^{2}\right] r d r, \\
F_{\mathrm{II}} & =\int_{0}^{1}\left[\left(\frac{1}{2} \sum_{k=1}^{\infty} \sum_{p=1}^{2} B_{k}^{(p)} n_{k}^{(p)}-n(r)\right)^{2}\right. \\
& \left.+\left(\frac{1}{2} \sum_{k=1}^{\infty} \sum_{p=1}^{2} B_{k}^{(p)} t_{k}^{(p)}-t(r)\right)^{2}\right] r d r, \\
F_{\mathrm{III}} & =\int_{0}^{1}\left[\left(\frac{1}{2} \sum_{k=1}^{\infty} \sum_{p=1}^{2} B_{k}^{(p)} \sigma_{k}^{(p)}-\sigma(r)\right)^{2}\right. \\
& \left.+\left(\frac{1}{2} \sum_{k=1}^{\infty} \sum_{p=1}^{2} B_{k}^{(p)} \tau_{k}^{(p)}-\tau(r)\right)^{2}\right] r d r . \\
& \left.+\left(\frac{1}{2} \sum_{k=1}^{\infty} \sum_{p=1}^{2} B_{k}^{(p)} t_{k}^{(p)}-t(r)\right)^{2}\right] r d r, \\
& =\int_{0}^{1}\left[\left(\frac{1}{2} \sum_{k=1}^{\infty} \sum_{p=1}^{2} B_{k}^{(p)} n_{k}^{(p)}-n(r)\right)^{2}\right.
\end{aligned}
$$

That reduces the problems to corresponding problems of unconstrained optimization.

Applying the necessary minimum conditions to the functionals (43) to (46)

$$
\begin{aligned}
& \frac{\partial F_{j}}{\partial B_{m}^{(1)}}=0, \\
& \frac{\partial F_{j}}{\partial B_{m}^{(2)}}=0,
\end{aligned}
$$

we come to the infinite system of linear algebraic equations

$$
\sum_{k=1}^{\infty} \sum_{p=1}^{2} M_{m k}^{(s p)} B_{k}^{(p)}=K_{m}^{(s)} .
$$


The coefficients $M_{m k}^{(s p)}(s, p=1,2, m=1,2, \ldots), K_{m}^{(s)}$ of system (48) for problems I' to IV' are defined by formulas (49)-(52) correspondingly:

$$
\begin{aligned}
M_{m k}^{(s p)}= & \frac{1}{2} \int_{0}^{1}\left(\sigma_{k}^{(p)} \sigma_{m}^{(s)}+\tau_{k}^{(p)} \tau_{m}^{(s)}\right) r d r, \\
K_{m}^{(s)}= & \int_{0}^{1}\left(\sigma(r) \sigma_{m}^{(s)}+\tau(r) \tau_{m}^{(s)}\right) r d r, \\
M_{m k}^{(s p)}= & \frac{1}{2} \int_{0}^{1}\left(n_{k}^{(p)} n_{m}^{(s)}+t_{k}^{(p)} t_{m}^{(s)}\right) r d r \\
& -\int_{0}^{1} n_{m}^{(s)} r d r \int_{0}^{1} n_{k}^{(p)} r d r, \\
K_{m}^{(s)}= & \int_{0}^{1}\left(n(r) n_{m}^{(s)}+t(r) t_{m}^{(s)}\right) r d r \\
& -2 \int_{0}^{1} n(r) r d r \int_{0}^{1} n_{m}^{(s)} r d r, \\
M_{m k}^{(s p)}= & \frac{1}{2} \int_{0}^{1}\left(\sigma_{k}^{(p)} \sigma_{m}^{(s)}+t_{k}^{(p)} t_{m}^{(s)}\right) r d r, \\
K_{m}^{(s)}= & \int_{0}^{1}\left(\sigma(r) \sigma_{m}^{(s)}+t(r) t_{m}^{(s)}\right) r d r, \\
M_{m k}^{(s p)}= & \frac{1}{2} \int_{0}^{1}\left(n_{k}^{(p)} n_{m}^{(s)}+\tau_{k}^{(p)} \tau_{m}^{(s)}\right) r d r \\
& -\int_{0}^{1} n_{m}^{(s)} r d r \int_{0}^{1} n_{k}^{(p)} r d r, \\
K_{m}^{(s)}= & \int_{0}^{1}\left(n(r) n_{m}^{(s)}+\tau(r) \tau_{m}^{(s)}\right) r d r \\
& -2 \int_{0}^{1} n(r) r d r \int_{0}^{1} n_{m}^{(s)} r d r .
\end{aligned}
$$

So, the problems are reduced to solving the infinite system of linear algebraic equations (48).

\section{Numerical Experiments}

We solved the problems with the use of the reduction method considering the finite system of dimension $2 N$ [19]:

$$
\sum_{k=1}^{N} \sum_{p=1}^{2} M_{m k}^{(s p)} B_{k}^{(p)}=K_{m}^{(s)} .
$$

To evaluate the convergence of the solutions for problems $I^{\prime}$ to $I V^{\prime}$ depending on dimension of the reduced system we considered some characteristic examples, taking the

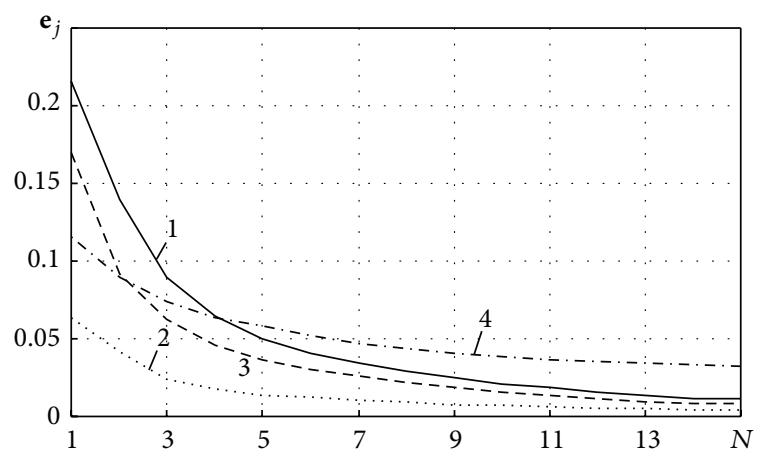

FIgURE 3: Solution error for problems I' to $\mathrm{IV}^{\prime}$.

functions of right-hand sides of boundary conditions (38)(41) in the forms

$$
\begin{aligned}
\sigma(r) & =\sigma_{0} \arctan \left(d\left(r-r_{0}\right)\right), \\
\tau(r) & =0, \\
r_{0} & =0.5, \\
d & =40, \\
n(r) & =0, \\
t(t) & =t_{0} r, \\
\sigma(r) & =\sigma_{0} \arctan \left(d\left(r-r_{0}\right)\right), \\
t(r) & =0, \\
n(r) & =0, \\
\tau(r) & =\tau_{0} r .
\end{aligned}
$$

The solution errors for problems I' to IV ${ }^{\prime}$ were calculated due to values of the corresponding functional as

$$
\begin{gathered}
\varepsilon_{\mathrm{I}}=\frac{1}{\sigma_{0}}\left(\frac{F_{\mathrm{I}}}{2 \pi}\right)^{1 / 2}, \\
\varepsilon_{\mathrm{II}}=\frac{1}{t_{0}}\left(\frac{F_{\mathrm{II}}}{2 \pi}\right)^{1 / 2}, \\
\varepsilon_{\mathrm{III}}=\frac{1}{\sigma_{0}}\left(\frac{F_{\mathrm{III}}}{2 \pi}\right)^{1 / 2}, \\
\varepsilon_{\mathrm{IV}}=\frac{1}{\tau_{0}}\left(\frac{F_{\mathrm{IV}}}{2 \pi}\right)^{1 / 2} .
\end{gathered}
$$

Plots in Figure 3 demonstrate how the errors $\mathbf{e}_{j} \in$ $\left\{\varepsilon_{\mathrm{I}}, \varepsilon_{\mathrm{II}}, \varepsilon_{\mathrm{III}}, \varepsilon_{\mathrm{IV}}\right\}$ decay with increasing $N$ (curves $1,2,3$, and 4 , resp.). As we can see convergence of the solutions depends on the type of boundary conditions and features of the prescribed boundary data. On the basis of conducted numerical experiments we can conclude that accuracy sufficient for practical goals can be achieved at $N \geq 10$.

In Figures 4-7 some results obtained by solving problems $\mathrm{I}^{\prime}$ and $\mathrm{II}^{\prime}$ are presented. Figure 4 displays the radial 


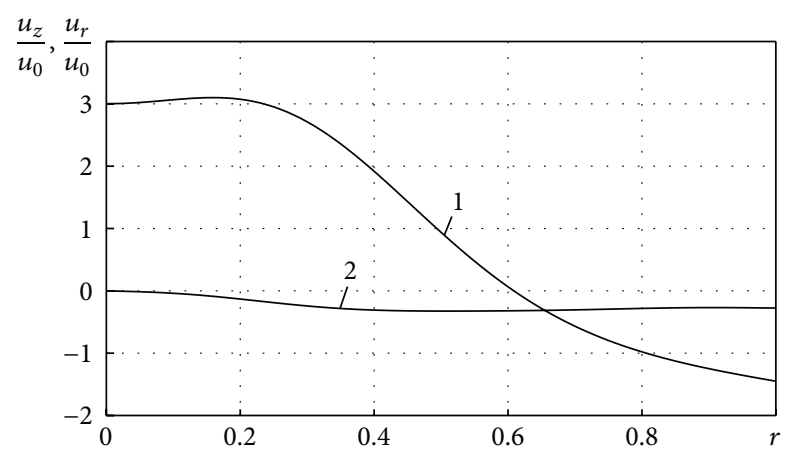

Figure 4: The radial dependencies of displacement for problem I'.

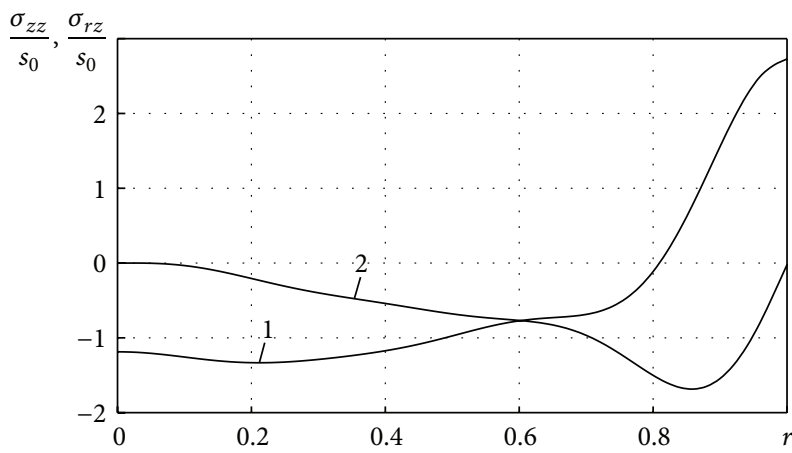

FIGURE 5: The radial dependencies of stresses for problem II'.

dependencies of dimensionless normal $u_{z} / u_{0}$ (curve 1) and tangential $u_{r} / u_{0}$ (curve 2) displacements on the surface $\mathbf{S} \subset$ $\partial \mathbf{V}$ for problem $I^{\prime}$. Figure 5 shows the radial dependencies of dimensionless normal $\sigma_{z z} / s_{0}$ (curve 1) and tangential $\sigma_{r z} / s_{0}$ (curve 2) stresses on this surface for problem $\mathrm{II}^{\prime}$.

Here $u_{0}$ and $s_{0}$ are parameters defined as $u_{0}=\sigma_{0} r_{0} /(2 \mu)$, $s_{0}=2 \mu t_{0} / r_{0}$, where $r_{0}=1$ is the cylinder radius.

Figures 6 and 7 illustrate axial dependences of dimensionless stress components for problems I' and $\mathrm{II}^{\prime}$ correspondingly.

Curves 1 and 2 in both figures correspond to the stress components $\sigma_{z z}$ calculated at constant radial coordinates $r=$ 0 and $r=1$, respectively, whereas curves 3 and 4 correspond to the stress components $\sigma_{\theta \theta}$ calculated at the same radial coordinates $r=0$ and $r=1$.

As we can see the solutions are quickly decayed with axial coordinate. So, developed approach can be applied to finite cylinders the heights of which equal two or more of their radii.

\section{Conclusion}

In this paper we proposed an approach for solving of the axisymmetric biharmonic boundary value problems for semi-infinite cylindrical domain, on the lateral surface of which homogeneous Neumann boundary conditions are prescribed. On the remining part of the domain's boundary four different biharmonic boundary pieces of data are considered. The approach is based on presentation of the

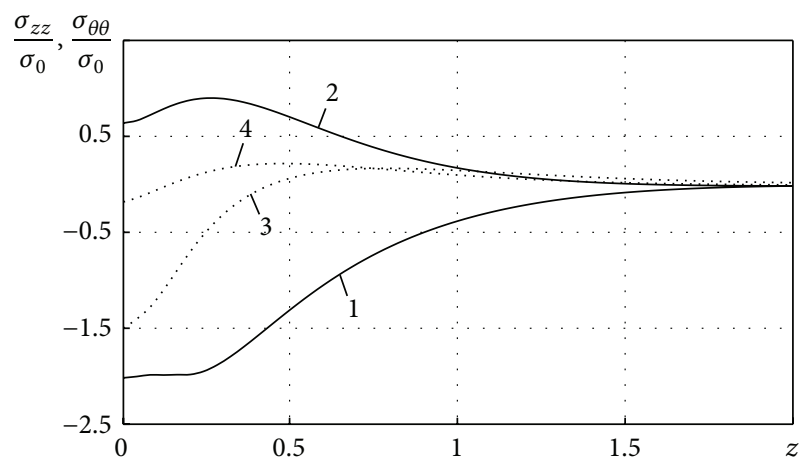

FIgURE 6: The axial dependencies of stress components for problem $I^{\prime}$.

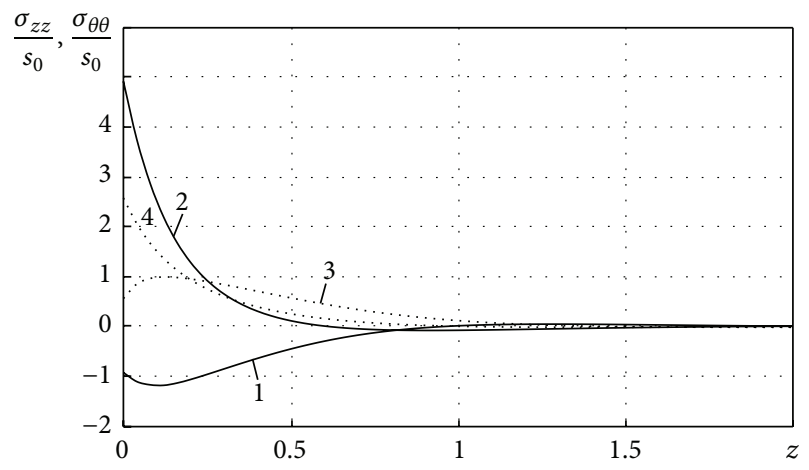

FIgURE 7: The axial dependencies of stress components for problem II'.

solution as a series expansion in two consequences of complex valued biharmonic functions, so-called homogeneous solutions, which obey the Neumann homogeneous boundary conditions on the lateral surface. Application of the method of least squares for subordinating the solution to nonhomogeneous boundary conditions prescribed on the part of the boundary reduces the problems to corresponding problems of nonconstrained optimization. These problems, in turn, were reduced to infinite systems of linear algebraic equations regarding the expansion coefficients. Solutions of the systems were obtained for various boundary data given on the part of the boundary with nonhomogeneous conditions exploiting the reduction method. Conducted numerical experiments confirm high convergence of the method: a sufficient accuracy is reached at $N$ equal to about 10 .

The approach can be used to solve axisymmetric elasticity problems for cylindrical bodies, the heights of which are equal to or exceed their diameters, when on their lateral surface normal and tangential tractions are prescribed and on the cylinder's end faces one of four possible boundary pieces of data is given: (1) normal and tangential tractions, (2) normal and tangential displacements, (3) normal traction and tangential displacement, and (4) normal displacement and tangential traction.

Similar approach can be developed for the case when on the cylinder's lateral surface boundary conditions in displacements are given. 
To expand the developed approach for a short cylindrical body, the height of which is less than its diameter, the interactions of strain-stressed states, caused by boundary data prescribed on the opposite end faces, should be accounted. To do that one can use four sequences $V^{(1)}, V^{(2)}, V^{(3)}$, and $V^{(4)}$ of homogeneous solutions to construct the solution. Here $V^{(3)}=\left\{v_{k}^{(3)}, k=1,2, \ldots\right\}, V^{(4)}=\left\{v_{k}^{(4)}, k=\right.$ $1,2, \ldots\}, v_{k}^{(3)}(r, z)$, and $v_{k}^{(4)}(r, z)$ are biharmonic functions corresponding transcendental equation (32) roots $\left\{\gamma_{k}^{(3)}, k=\right.$ $1,2, \ldots\}$ and $\left\{\gamma_{k}^{(4)}, k=1,2, \ldots\right\}$.

\section{Competing Interests}

The authors declare that they have no competing interests.

\section{References}

[1] H. Begehr, "Dirichlet problems for the biharmonic equation," General Mathematics, vol. 13, no. 2, pp. 65-72, 2005.

[2] Q. A. Dang and N. Van Thien, "Iterative method for solving the second boundary value problem for biharmonic-type equation," Journal of Applied Mathematics, vol. 2012, Article ID 891519, 18 pages, 2012.

[3] B. Turmetov and R. Ashurov, "On solvability of the Neumann boundary value problem for non-homogeneous biharmonic equation," British Journal of Mathematics \& Computer Science, no. 4, pp. 557-571, 2014.

[4] V. Moises, Mixed problems and layer potentials for harmonic and biharmonic functions [thesis], Syracuse University, Syracuse, NY, USA, paper 66, 2011.

[5] O. A. Matevosyan, "Solution of a mixed boundary value problem for the biharmonic equation with finite weighted Dirichlet integral," Differential Equations, vol. 51, no. 4, pp. 487-501, 2015.

[6] Q. A. Dang, "Iterative method for solving the Neumann boundary value problem for biharmonic type equation," Journal of Computational and Applied Mathematics, vol. 196, no. 2, pp. 634-643, 2006.

[7] S. Jiang, B. Ren, P. Tsuji, and L. Ying, "Second kind integral equations for the first kind Dirichlet problem of the biharmonic equation in three dimensions," Journal of Computational Physics, vol. 230, no. 19, pp. 7488-7501, 2011.

[8] M. Ben-Artzi, I. Chorev, J.-P. Croisille, and D. Fishelov, "A compact difference scheme for the biharmonic equation in planar irregular domains," SIAM Journal on Numerical Analysis, vol. 47, no. 4, pp. 3087-3108, 2009.

[9] J. Pestana, R. Muddle, M. Heil, F. Tisseur, and M. Mihajlović, "Efficient block preconditioning for a $\mathrm{C}^{1}$ finite element discretization of the dirichlet biharmonic problem," SIAM Journal on Scientific Computing, vol. 38, no. 1, pp. A325-A345, 2016.

[10] A. I. Lurie, Theory of Elasticity, Springer, Berlin, Germany, 2005.

[11] S. A. Lurie and V. V. Vasiliev, The Biharmonic Problem in The Theory of Elasticity, Gordon and Breach Publishers, Amsterdam, The Netherlands, 1995.

[12] V. V. Meleshko, "Biharmonic problem in a rectangle," Applied Scientific Research, vol. 58, no. 1-4, pp. 217-249, 1997.

[13] V. T. Grinchenko, "The biharmonic problem and progress in the development of analytical methods for the solution of boundary-value problems," Journal of Engineering Mathematics, vol. 46, no. 3-4, pp. 281-297, 2003.
[14] F. Chekurin and L. I. Postolaki, "A variational method for the solution of biharmonic problems for a rectangular domain," Journal of Mathematical Sciences, vol. 160, no. 3, pp. 386-399, 2009.

[15] K. Rektorys and V. Zahradník, "Solution of the first biharmonic problem by the method of least squares on the boundary," Aplikace Matematiky, vol. 19, no. 2, pp. 101-131, 1974.

[16] R. Sburlati, "Three-dimensional analytical solution for an axisymmetric biharmonic problem," Journal of Elasticity, vol. 95, no. 1, pp. 79-97, 2009.

[17] G. Fairweather, A. Karageorghis, and Y.-S. Smyrlis, "A matrix decomposition MFS algorithm for axisymmetric biharmonic problems," Advances in Computational Mathematics, vol. 23, no. 1-2, pp. 55-71, 2005.

[18] W. Nowacki, The Theory of Elasticity, Mir, Moscow, Russia, 1975 (Russian).

[19] I. V. Kantorovich and V. I. Krylov, Approximate Methods of Higher Analysis, Phys.-Mat. Publ., Moscow, Russia, 1962 (Russian), Translated from the 3rd Russian edition by C. D. Benster, Interscience Publishers, New York, NY, USA; P. Noordhoff, Groningen, The Netherlands, 1958. 


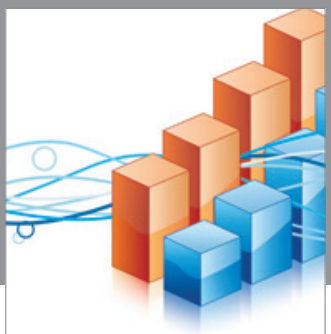

Advances in

Operations Research

vatem alat4

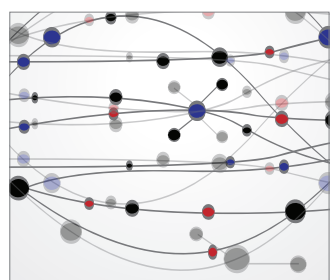

\section{The Scientific} World Journal
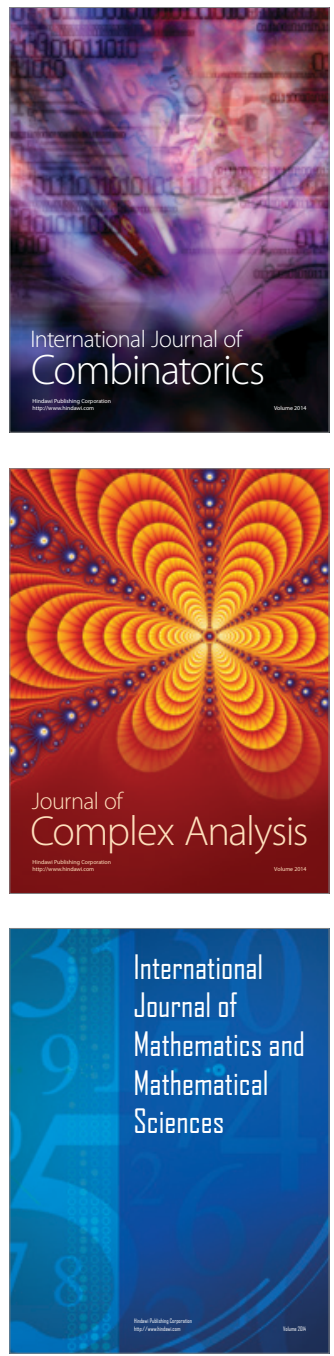
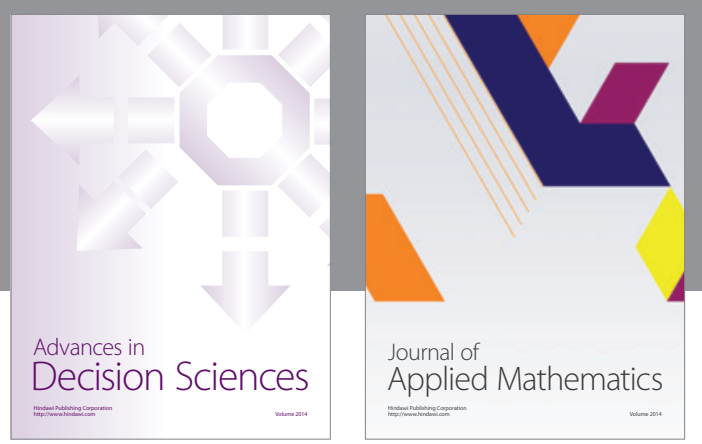

Algebra

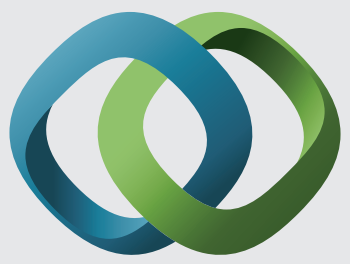

\section{Hindawi}

Submit your manuscripts at

http://www.hindawi.com
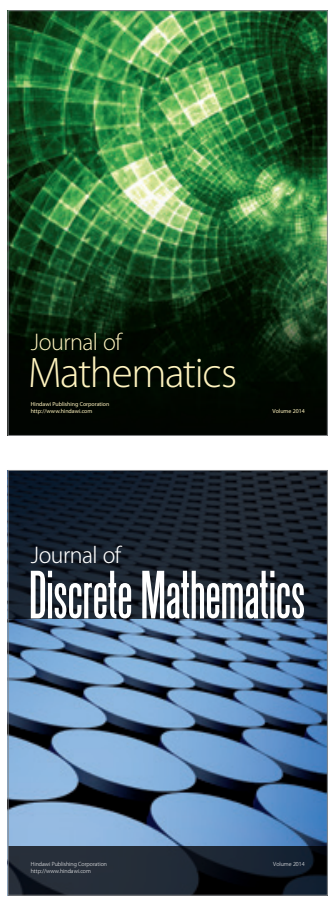

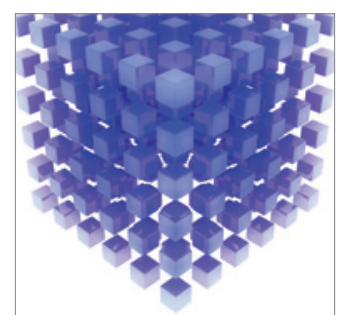

Mathematical Problems in Engineering
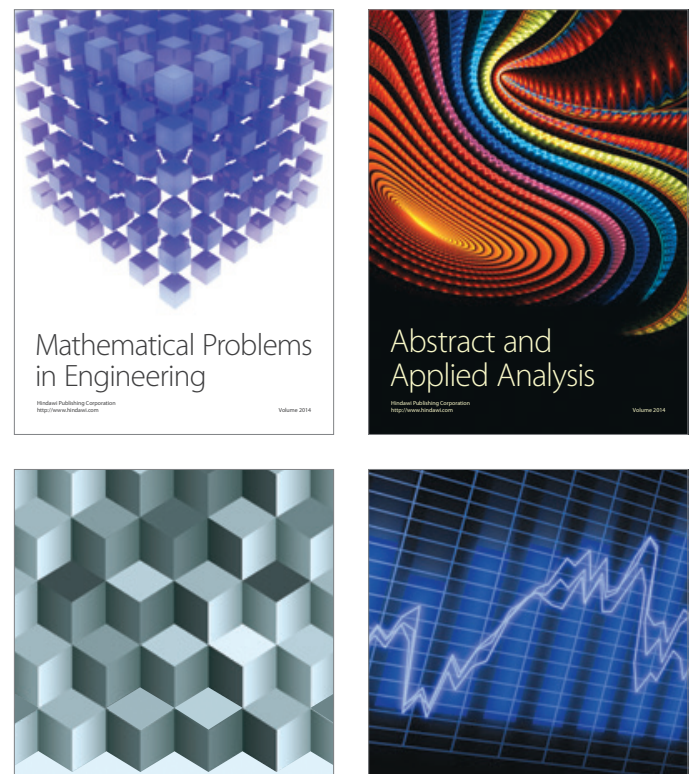

Journal of

Function Spaces

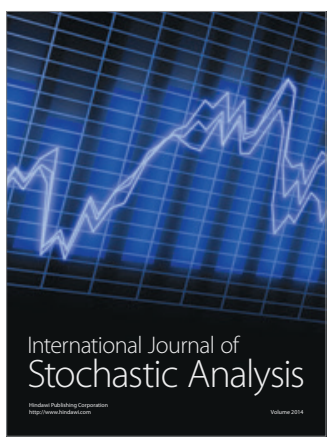

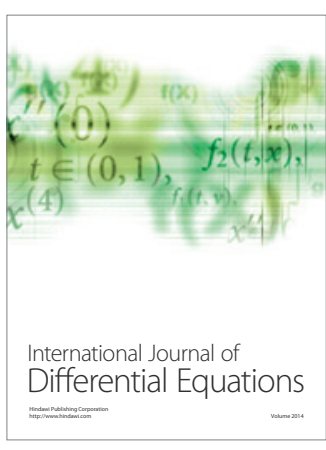
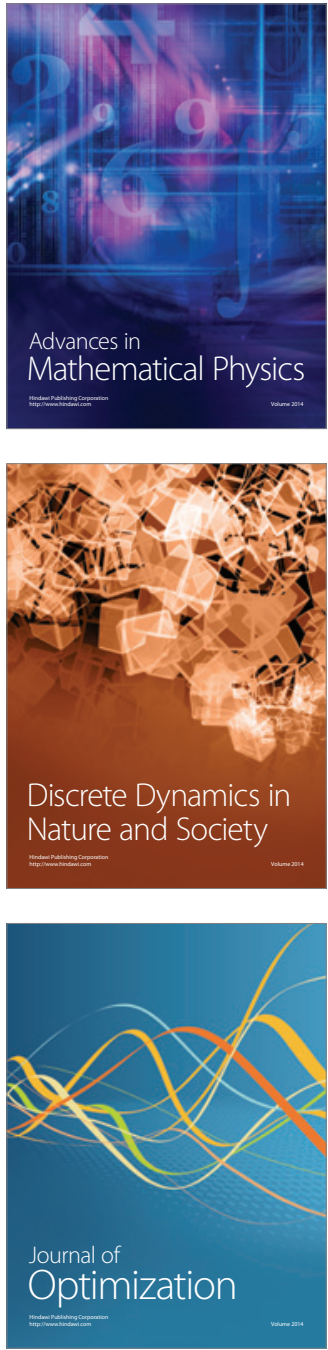